\title{
Efeito hipoglicemiante da farinha do fruto de maracujá-do-mato (Passiflora nitida Kunth) em ratos normais e diabéticos
}

\author{
LIMA, E.S. ${ }^{1 *}$; SCHWERTZ, M.C. ${ }^{2}$; SOBREIRA, C.R.C. ${ }^{1}$, BORRAS, M.R.L.' \\ ${ }^{1}$ Faculdade de Ciências Farmacêuticas, Universidade Federal do Amazonas - UFAM, Rua Alexandre Amorim, 330, \\ CEP: 69010-300, Bairro Aparecida, Manaus-Brasil *eslima@ufam.edu.br ${ }^{2}$ Instituto Nacional de Pesquisas da \\ Amazônia, Coordenação de Pesquisas em Ciências da Saúde, Avenida André Araújo, 2936, Petrópolis, CEP: \\ 69083-000, Manaus-Brasil
}

\begin{abstract}
RESUMO: A região amazônica é detentora de uma vasta biodiversidade de frutos, porém pouco explorada quanto o seu potencial nutricional e econômico. Dentre estes frutos destaca-se o maracujá-do-mato (Passiflora nitida Kunth), espécie silvestre, de fruto comestível, com sabor exótico e de boa aceitabilidade para consumo. No presente estudo objetivou-se analisar as características nutricionais do mesocarpo do fruto da $P$. nitida e avaliar o potencial hipoglicemiante em ratos normais e diabéticos. A farinha do mesocarpo do fruto foi elaborada e analisada quanto a composição centesimal. A atividade hipoglicemiante foi avaliada por meio de dois modelos experimentais em ratos Wistar. O mesocarpo apresentou baixa concentração de macronutrientes e alto teor de umidade, cinzas e fibras. No experimento agudo, após 15 minutos da administração da sacarose, os níveis glicêmicos foram de $146 \pm 12 \mathrm{mg} \mathrm{dL}^{-1}$ no grupo controle e $112 \pm 2,5 \mathrm{mg} \mathrm{dL}^{-1}$, no grupo que recebeu $1 \mathrm{~g} \mathrm{~kg}^{-1}$ de peso da farinha. No experimento crônico, após 21 dias, houve redução de $493 \mathrm{mg} \mathrm{dL}^{-1}$ para $302 \mathrm{mg} \mathrm{dL}^{-1}$ (38,7\%) e $195 \mathrm{mg} \mathrm{dL}^{-1}(60,4 \%)$ na glicemia nos grupos que foram tratados com 20 e $40 \%$ de ração enriquecida com a farinha, respectivamente, em relação ao grupo diabético não tratado. Em ambos os modelos experimentais, a farinha do mesocarpo mostrou-se eficaz na redução da glicemia. $O$ fruto de $P$. nitida mostrou-se um produto natural em potencial para o controle da glicemia no diabetes.
\end{abstract}

Palavras-chave: Maracujá-do-mato, Passiflora nitida, diabetes, atividade hipoglicemiante

\begin{abstract}
Hypoglycemic effect of Passiflora nitida Kunth fruit flour on normal and diabetic rats. The Amazon region has a vast biodiversity of fruits but is little explored as to its nutritional and economic potential. Among these fruits is "maracuja-do-mato" (Passiflora nitida Kunth), a wild species of edible fruit with exotic flavor and good acceptability for consumption. The aim of the present study was to analyze the nutritional characteristics of $P$. nitida fruit mesocarp and to evaluate its hypoglycemic potential in normal and diabetic rats. Flour from the fruit mesocarp was prepared and analyzed as to its centesimal composition. Hypoglycemic activity was assessed by means of two experimental models in Wistar rats. The mesocarp showed low concentration of macronutrients and high humidity, ash and fiber content. In the acute experiment, after 15 minutes of sucrose administration, glucose levels were $146 \pm 12 \mathrm{mg} \mathrm{dL}^{-1}$ in the control group and $112 \pm 2.5 \mathrm{mg}$ $\mathrm{dL}^{-1}$ in the group receiving $1 \mathrm{~g} \mathrm{~kg}^{-1}$ flour weight. In the chronic experiment, after 21 days, glucose levels reduced from $493 \mathrm{mg} \mathrm{dL}^{-1}$ to $302 \mathrm{mg} \mathrm{dL}^{-1}(38.7 \%)$ and $195 \mathrm{mg} \mathrm{dL}^{-1}(60.4 \%)$ in the groups treated with 20 and $40 \%$ animal food enriched with the flour, respectively, in relation to the diabetic untreated group. In both experimental models, the mesocarp flour was effective in reducing blood glucose. P. nitida fruit seems to be a potential natural product in the glycemic control of diabetes.
\end{abstract}

Key words: "maracujá-do-mato", Passiflora nitida, diabetes, hypoglycemic effect

\section{INTRODUÇÃO}

O diabetes melittus do tipo 2 é uma doença crônica, de origem endócrina, caracterizada pela hiperglicemia e graus variados de resistência a insulina (incapacidade da insulina de exercer seus efeitos nos tecidos-alvo). É um problema de saúde pública que já atinge 171 milhões de indivíduos em todo mundo e com projeção de alcançar 370 milhões de pessoas no ano de 2030 (Agra et al., 2007). Segundo

Recebido para publicação em 05/07/2011

Aceito para publicação em 20/03/2012

Rev. Bras. PI. Med., Botucatu, v.14, n.2, p.383-388, 2012. 
informações do Sistema Único de Saúde do Brasil, o diabetes aparece como a sexta causa primária de internações hospitalares e contribui de forma significativa (30 a 50\%) para outras causas de internação hospitalar (Silva et al., 2010). Reduzir o impacto do diabetes significa, antes de tudo, reduzir a incidência da doença, antecipando-se ao aparecimento com medidas preventivas, como por exemplo, por meio de cuidados dietéticos, sobretudo em indivíduos de alto risco, ou ainda, controlar os sintomas evitando futuras consequências ou agravos da doença (Wild et al., 2004).

A busca por produtos naturais para prevenção e tratamento de doenças, como o diabetes, vem crescendo a cada dia, o que tem impulsionado as pesquisas referentes ao potencial funcional de muitos alimentos. Pois além dos aspectos nutricionais, os mesmos também apresentam efeitos fisiológicos e metabólicos benéficos à saúde, como por exemplo, alimentos que previnam a hiperglicemia ou melhorem a tolerância à glicose em indivíduos saudáveis e diabéticos (Derivi et al., 2002).

$\mathrm{Na}$ vasta biodiversidade amazônica destaca-se o maracujá-do-mato (Passiflora nitida Kunth), da família Passifloraceae, com fruto silvestre, comestível, de sabor exótico e boa aceitabilidade para consumo. O fruto in natura é bastante apreciado por populações do norte do Brasil. Este fruto apresenta componentes com potencial funcional como fibras, vitaminas, carotenóides e componentes inorgânicos (cálcio, ferro, fósforo) flavonóides, esteróides e ácidos graxos) (Moraes et al., 2002). Diversos usos alimentares e terapêuticos já foram descritos para algumas espécies do gênero Passiflora, com destaque para o maracujáamarelo ou azedo (Passiflora edulis f. flavicarpa Degener). A casca e o mesocarpo de outra espécie do gênero Passiflora, a $P$. edulis, tem sido utilizada como auxiliar no tratamento do diabetes, devido seu alto teor de fibras, principalmente de fibra solúvel, pectina as quais tem se mostrado efetivas como adjuvantes no tratamento do diabetes (Agra et al., 2007; Krahn et al., 2008; Weickert \& Pfeiffer 2008).

Até o presente momento a espécie $P$. nitida foi pouco explorada do ponto de vista científico e biotecnológico. Carvalho e colaboradores (2010) relataram atividades antioxidante, anti-coagulante e anti-agregante plaquetária de extratos das folhas desta espécie. Neste sentido, objetivou-se avaliar o papel na absorção de carboidratos e o potencial hipoglicemiante da farinha do mesocarpo do fruto do maracujá-do-mato em ratos normais e diabéticos, respectivamente.

\section{MATERIAL E MÉTODO}

\section{Obtenção da farinha}

Frutos de $P$. nitida, originários do município de Rio Preto da Eva, Amazonas, foram adquiridos no comércio da cidade de Manaus em estágio de maturação. Foram submetidos aos processos de seleção, sanitização, despolpe e descasca. Obtendose apenas o mesocarpo. O mesocarpo foi desidratado a 55ํㅡ em estufa com circulação de ar forçada por $15 \mathrm{~h}$, até peso constante. Em seguida, o mesocarpo seco foi triturado em liquidificador doméstico e peneirado para obtenção da farinha. A farinha foi embalada em sacos de polietileno e armazenada em geladeira até a utilização. Foram retiradas três alíquotas da farinha para determinação da composição nutricional.

\section{Composição centesimal}

As determinações de umidade, cinzas, lipídeos, proteínas e glicídeos totais do mesocarpo fresco de $P$. nitida foram realizadas segundo métodos analíticos do Instituto Adolfo Lutz (IAL, 1985) e da Association of Official Analytical Chemistry (AOAC, 2000). Os teores de fibra bruta foram analisados por digestão ácida em solução de ácido sulfúrico 1,25\%, segundo Moretto et al., (2002). O valor calórico da amostra foi calculado pela soma do percentual de proteína bruta e glicídios, multiplicado pelo fator 4 (Kcal $\left.\mathrm{g}^{-1}\right)$, somando o teor de lipídios totais, multiplicando pelo fator 9 (Kcal g$\left.{ }^{-1}\right)$ (Maihara, et al., 2006).

\section{Animais}

Foram utilizados ratos machos, adultos, da linhagem Wistar (Rattus norvegicus var. albinus Berkenhout), com 60 dias de vida e peso aproximado de $150 \mathrm{~g}$, provenientes do Biotério Central da UFAM. Os animais foram mantidos em temperatura de $22 \pm 2^{\circ} \mathrm{C}$ e umidade de $60 \pm 10 \%$, com ciclo claro-escuro de 12 horas, em número de cinco animais por gaiolas de polietileno, recebendo água e ração comercial (Labina ${ }^{\circledR}$, purina) ad libitum, exceto 12 horas antes dos experimentos, os quais foram realizados com os animais em jejum. Todas as experimentações com animais e os procedimentos realizados estiveram de acordo com aqueles preconizados pela Lei $n^{\circ} 11.794$ de 2008, que regulamenta a utilização de animais com fins científicos no Brasil. O projeto foi aprovado pelo Comitê de Ética de Pesquisa Animal da UFAM (№23105.003147/2010).

\section{Atividade hipoglicemiante em ratos normais}

Foram utilizados 15 ratos, divididos aleatoriamente em quatro grupos: Grupo I-controle normal que recebeu apenas água ad libitum $(n=5)$ e Grupos II e III tratados com a farinha de P. nitida em

Rev. Bras. PI. Med., Botucatu, v.14, n.2, p.383-388, 2012. 
suspensão aquosa nas doses de 0,5 e 1,0 $\mathrm{g} \mathrm{Kg}^{-1}$ de peso, respectivamente ( $n=5$, cada) e grupo IV que recebeu uma dose de $50 \mathrm{mg} \mathrm{kg}^{-1}$ de peso de acarbose (Sigma-Aldrich, Sto. Luis, USA) como controle positivo para o teste. Após $12 \mathrm{~h}$ de jejum, os animais do grupo I receberam a água, dos grupos II e III a farinha nas respectivas doses e o grupo IV recebeu acarbose. Após $1 \mathrm{~h}$ da administração da farinha, os animais de todos os grupos receberam $1,5 \mathrm{~g} \mathrm{Kg}^{-1}$ de sacarose em solução aquosa. A água e as soluções administradas aos animais foram realizadas por via intragástrica. Amostras de sangue foram coletadas da veia caudal nos tempos de 0,15, 30, 60, 90 e 120 min após a sobrecarga de sacarose. A glicemia foi mensurada com glicosímetro ACCU-CHEK ${ }^{\circledast}$ (Soares et al., 2000).

Atividade hipoglicemiante em ratos diabéticos

$O$ diabetes experimental foi induzido por única injeção intraperitoneal de $150 \mathrm{mg} \mathrm{Kg}^{-1}$ de aloxano monohidratado (Sigma-Aldrich, St. Louis, EUA), diluído a $2 \%$ em solução de citrato de sódio 0,05M, pH 4,5, após jejum de 24 h (Almeida et al., 2006). No terceiro dia após a injeção de aloxano e monitoramento dos níveis basais de glicemia, os animais diabéticos (com glicemia $>250 \mathrm{mg} \mathrm{dL}^{-1}$ ) foram separados e divididos aleatoriamente em três grupos: Grupo I-diabético que recebeu $10 \mathrm{~mL} \mathrm{Kg}^{-1}$ de água destilada ( $n=5)$; Grupos II e III-diabéticos tratados com $20 \%$ e $40 \% \mathrm{p} / \mathrm{p}$, respectivamente, da farinha do maracujá-do-mato incorporada a ração comercial (Labina ${ }^{\circledR}$, Campinas, SP) ( $n=5$, cada). Os animais foram mantidos com essa dieta, nas condições experimentais previamente descritas por 21 dias. Amostras de sangue foram coletadas através da veia caudal nos dias 1, 3, 6, 9, 12, 15, 18 e 21, após o início da introdução da farinha na dieta. Os níveis de glicose no sangue foram determinados com glicosímetro ACCU-CHEK ${ }^{\circledast}$ (Soares et al., 2000).

\section{Análise estatística}

Os resultados obtidos foram analisados pelo programa SigmaStat para Windows, versão 2.0 (Jandel, San Rafael, CA, EUA) e apresentados na forma gráfica por utilização do Programa Excel (Microsoft Word., St.Louis, MO, EUA). Foi utilizado o teste de Kolmogorov-Smirnov para analisar a distribuição dos dados. Para comparação entres os diferentes grupos foi utilizado o teste de variância ANOVA $(p<0,05)$.

\section{RESULTADO E DISCUSSÃO}

Os resultados da análise centesimal do mesocarpo fresco de $P$. nitida estão apresentados na Tabela 1. O mesocarpo do fruto apresentou teor de umidade de aproximadamente $80 \%$, o que é esperado em se tratando de parte de fruto fresco.
Em relação aos macronutrientes as concentrações não foram elevadas onde a amostra demonstrou teor de aproximadamente $0,03 \%$ para glicídios e $1 \%$ para proteínas. Estes dados indicam que se trata de um produto com baixo valor calórico, uma vez que no calculo das calorias o valor encontrado foi de

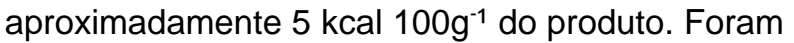
encontradas concentrações de minerais totais (cinzas) em torno de 3\%, o que é relativamente normal em se tratando de fruto. A concentração de fibra bruta encontrada $(14,6 \pm 0,08 \%)$ é relativamente alta, quando comparada com aquelas encontradas em outros frutos.

Estes resultados, por exemplo, diferem dos encontrados por Collazos (1975), que verificou concentrações menores desses constituintes no fruto de maracujá-do-mato. Uma hipótese que pode ser considerada para essas diferenças são variações genéticas, edafo-climáticas ou sazonais que podem interferir na composição do fruto. No entanto, resultados semelhantes foram encontrados por Gondim et al. (2005), ao analisarem a farinha do maracujá-amarelo ( $P$. edulis).

O teor de fibra de aproximadamente $14 \%$ encontrada no mesocarpo fresco do fruto de $P$. nitida, propícia a utilização no planejamento dietético de indivíduos saudáveis ou diabéticos para reduzir ou retardar a absorção de glicose. O emprego do mesocarpo do fruto na forma de farinha apresenta algumas vantagens como a concentração das fibras ao retirar a umidade, aumento da vida de prateleira do alimento, maior facilidade no armazenamento e na viabilização do emprego na elaboração de produtos derivados.

A atividade hipoglicemiante no ensaio agudo em ratos normoglicêmicos foi expressa pela redução da glicemia medida nos tempos $0,15,30,60$, e 120 minutos, pós-sobrecarga de $1,5 \mathrm{~g} \mathrm{~kg}^{-1}$ de peso de uma solução de sacarose. Os valores médios de glicemia no tempo zero não apresentaram diferença significativa entre os grupos (Figura 1). Porém nos demais tempos, houve aumento significativo da

TABELA 1. Composição centesimal do mesocarpo fresco do fruto de $P$. nitida.

\begin{tabular}{lc}
\hline Parâmetro & Media \pm D.P. \\
\hline Umidade $(\mathrm{g} \%)$ & $81,1 \pm 0,273$ \\
Proteínas $(\mathrm{g} \%)$ & $1,0 \pm 0,002$ \\
Lipídios $(\mathrm{g} \%)$ & $0,1 \pm 0,006$ \\
Cinzas $(\mathrm{g} \%)$ & $3,1 \pm 0,037$ \\
Fibra bruta $(\mathrm{g} \%)$ & $14,6 \pm 0,080$ \\
Carboidratos $(\mathrm{g} \%)$ & 0,034 \\
Energia (kcal $\left.100 \mathrm{~g}^{-1}\right)$ & 5,144 \\
\hline
\end{tabular}


glicemia no grupo I (não tratado) e uma resposta hipoglicemiante dose-dependente nos intervalos de 15,30 e 60 minutos nos grupos II e III e no grupo tratado com acarbose que apenas não apresentou redução significativa da glicemia no tempo de 60 minutos. Observou-se neste teste que houve uma diminuição significativa da glicemia, que chegou a $60 \%$ nos grupos tratados com a farinha de $P$. nitida (1 $\mathrm{g} \mathrm{kg}^{-1}$ de peso) ou acarbose, 15 minutos após administração da sacarose. É importante salientar que houve diferença estatística entre as glicemias dos grupos tratados e controle nos tempos 15, 30 e 60 minutos e como esperado em 120 minutos, houve retorno dos níveis glicêmicos aos níveis basais de glicose.

Apesar de eficazes, as doses administradas da farinha (0,5 e $1 \mathrm{~g} \mathrm{~kg}^{-1}$ de peso) foram relativamente altas, por exemplo, quando comparamos as doses eficazes de outros bioativos como extratos vegetais e drogas hipoglicemiantes, mas que agem por outros mecanismos de ação (Soares et al., 2000). Contudo, salienta-se que as mesmas foram bastante toleradas pelos animais, os quais não demonstraram nenhum sinal ou sintoma de desconforto ou toxicidade como irritação ou diarréia.

O método adotado para a indução do diabetes para avaliação do efeito hipoglicemiante em ratos foi eficaz, uma vez que a maioria dos animais induzidos com aloxano apresentaram glicemia superior à 250 $\mathrm{mg} \mathrm{dL}^{-1}$ no terceiro dia com baixo índice de mortalidade. As dosagens glicêmicas obtidas aproximam-se dos valores descritos na literatura por autores que utilizaram o mesmo método de indução. Neste caso, salienta-se que o efeito tóxico do aloxano se dá por destruição das células beta pancreáticas, reduzindo com isso drasticamente os níveis de insulina. Por este motivo, os níveis glicêmicos são tão elevados e consequentemente bem difíceis de serem reduzidos a níveis normais sem administração de insulina exógena (Schoenfelder et al., 2006).

Conforme apresentado na Figura 2, no experimento com animais diabéticos, pôde-se observar que a ingestão da farinha do mesocarpo de $P$. nitida produziu significativo efeito hipoglicemiante a partir do terceiro dia de tratamento quando a ração foi enriquecida com $40 \%$ de farinha (Grupo II) quando comparada com o grupo controle (diabéticos sem tratamento). Este efeito hipoglicemiante mais demorado pode estar associado à formação do filme gelificado, formado pelas fibras solúveis no lúmen intestinal (Córdova et al., 2005). Como descrito na literatura, a provável ação hipoglicemiante dos resíduos das espécies de Passiflora pode ser atribuída à presença das fibras, as quais formariam um gel na parede intestinal alterando a absorção de glicose e melhorando a tolerância a mesma, proporcionando um decréscimo na absorção sérica de glicose em dietas ricas em carboidratos (Córdova et al., 2005).

No experimento crônico, quando ao controle do peso, ingestão de ração e água pelos animais, não houve diferenças significativas entre os grupos tratados com a farinha de $P$. nitida. Não houve diferença estatisticamente significativa quanto ao consumo de líquido do grupo II, quando comparado

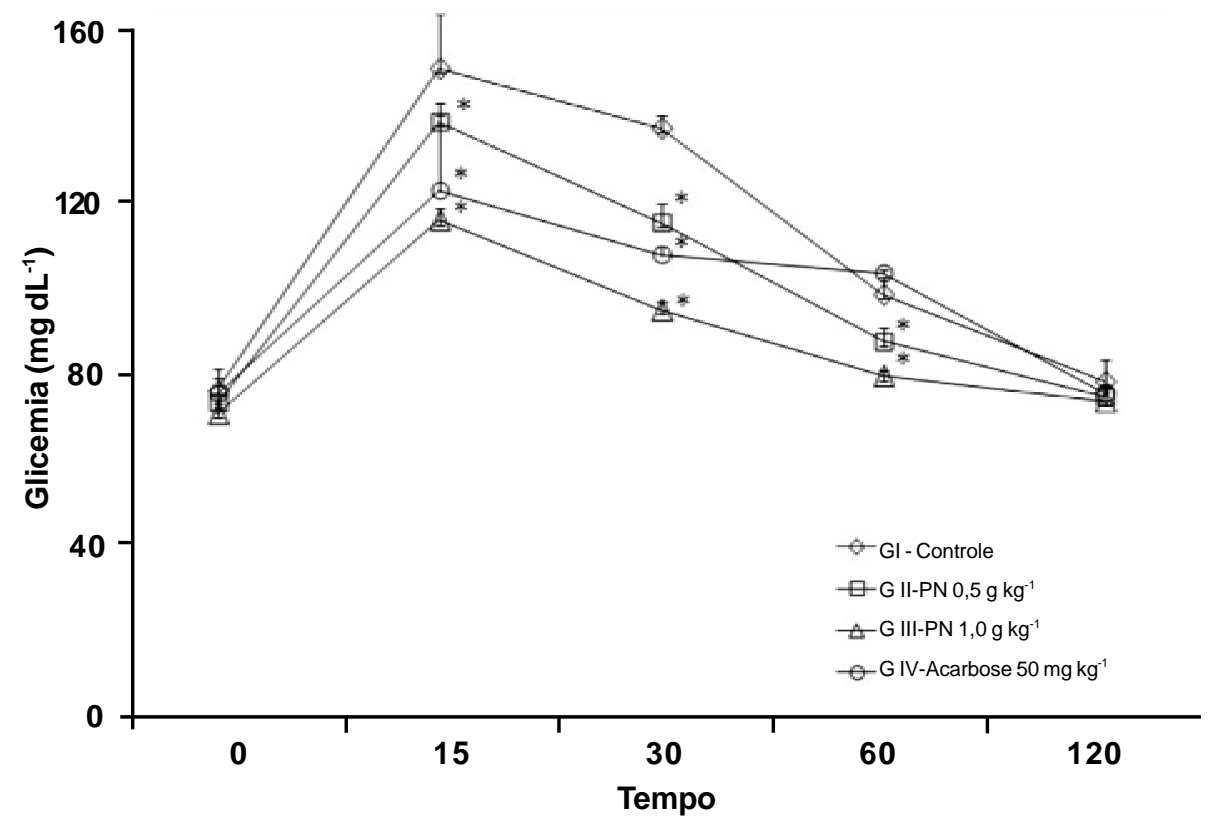

FIGURA 1. Efeito da administração oral da farinha do mesocarpo de $P$. nitida ou acarbose sobre a glicemia de ratos normais ( $n=5$, por grupo) pós-sobrecarga de sacarose. ${ }^{*}$ Estatisticamente diferente do grupo controle $(p<0,05$, ANOVA). 


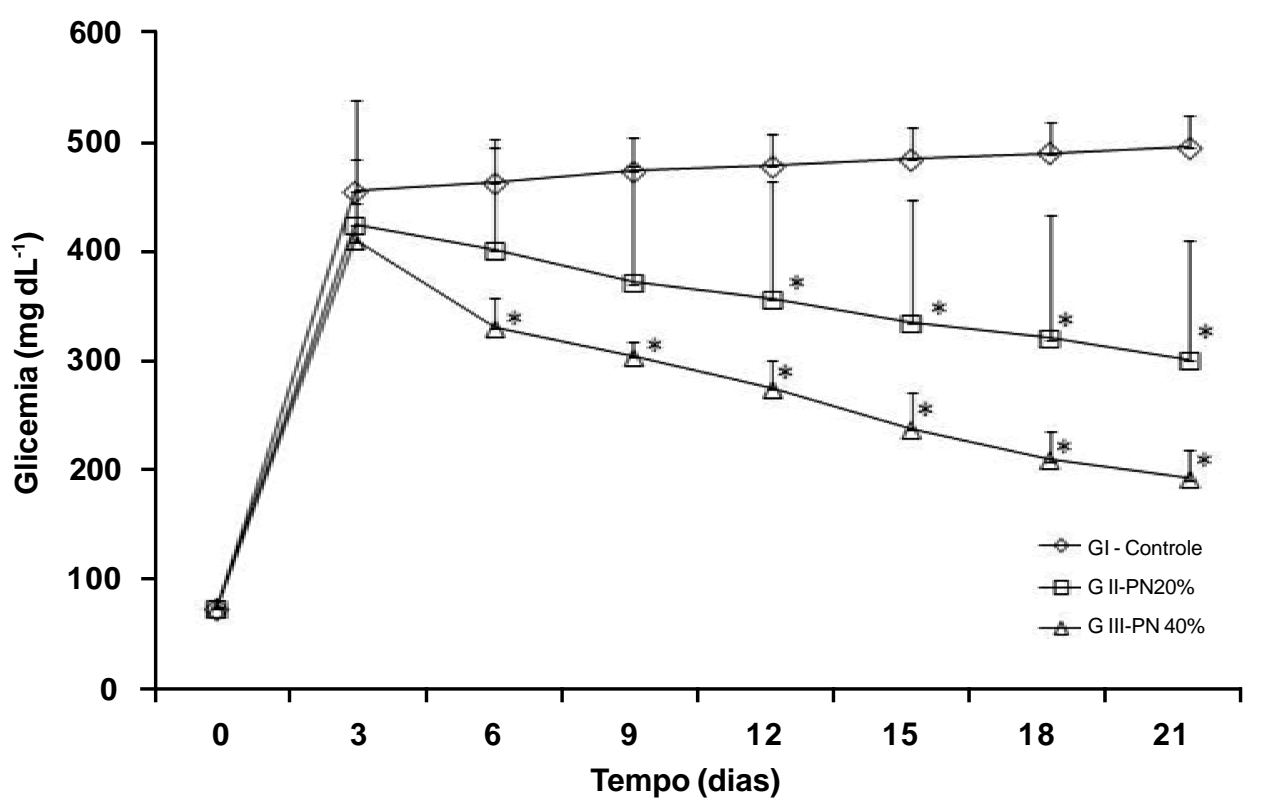

FIGURA 2. Efeito hipoglicemiante em ratos diabéticos ( $n=5$ por grupo) tratados ou não (grupo controle) durante 21 dias com farinha do mesocarpo do $P$. nitida incorporada em ração comercial triturada, nas concentrações de $20 \mathrm{e}$ $40 \%$. ${ }^{*}$ Estatisticamente diferente do grupo controle $(p<0,05$, ANOVA).

com o grupo I. Contudo, quando o grupo diabético sem tratamento é comparado com os grupos I e II observou-se uma redução gradativa da ingestão de água. No vigésimo primeiro dia do experimento, a média de consumo de cada grupo foi $99 \mathrm{~mL} /$ animal/ dia para o grupo controle, $57 \mathrm{~mL} /$ animal/dia para o grupo l e $31 \mathrm{~mL} /$ animal/dia para o grupo II. Quanto ao controle do peso dos animais, o grupo controle (diabéticos sem tratamento) aumentaram de peso entre $01^{\circ}$ e $21^{\circ}$ dia do experimento $(235 \pm 17 \mathrm{~g} \mathrm{e}$ $248 \pm 16 \mathrm{~g}$, respectivamente).

Nos grupos tratados com a farinha houve diminuição do peso quando comparado $01^{\circ}$ e o $021^{\circ}$ dia do experimento. No grupo I os animais foram de $259 \pm 16 \mathrm{~g}$ a $244 \pm 23 \mathrm{~g}$ e no grupo Il a variação de peso foi de $232 \pm 31 \mathrm{~g}$ a $216 \pm 29 \mathrm{~g}$. O aumento de peso nos animais diabéticos e a redução de peso nos animais tratados, pode está relacionado diretamente a diminuição da ingestão de água observada no presente trabalho e a consequente retenção de água que ocorre no diabetes o que é já relatada na literatura (Krahn et al., 2008).

Ainda no experimento com animais diabéticos, o grupo II apresentou diminuição significativa da glicemia a partir do sexto dia do experimento, enquanto que o grupo I, que recebeu a ração enriquecida com $10 \%$ de farinha, só apresentou diminuição significativa a partir do décimo segundo dia do experimento, conforme demonstrado na Figura 2. Ao final do experimento (21 dias) houve uma diminuição significativa da glicemia no grupo II $(p<$ 0,05 ) e uma redução mais acentuada no grupo II
( $p<0,001)$, quando comparado com o grupo diabético sem tratamento. Houve diferença significativa entre as diferentes doses do tratamento onde o grupo que recebeu dieta enriquecida com $40 \%$ da farinha mostrou redução mais significativa da glicemia no período.

O efeito hipoglicemiante da farinha do mesocarpo de $P$. nítida pode também está relacionado ao mecanismo que estudos recentes têm evidenciado. Fibras alimentares melhoram o metabolismo de carboidratos simples, apresentando uma relação inversa com o diabetes (Mira et al., 2009). As fibras alimentares podem também auxiliar no controle glicêmico de pacientes diabéticos (Weickert \& Pfeiffer, 2008). Segundo Howarth (2001) os benefícios das fibras são obtidos por meio de ingestão, acima de $25 \mathrm{~g}$ dia $^{-1}$ para mulheres e $38 \mathrm{~g} \mathrm{dia}^{-1}$ para homens. O aumento da ingestão de fibras totais também está inversamente associado com os marcadores de resistência à insulina (Pi-Sunyer, 2005; Bulló et al., 2007).

\section{CONCLUSÃO}

Os resultados encontrados evidenciaram pela primeira vez que a farinha do mesocarpo desidratado de $P$. nitida possui efeito inibitório sobre a absorção de sacarose em ratos normais e efeito hipoglicemiante em ratos diabéticos induzidos por aloxano. Neste sentido, o estudo evidenciou claramente o efeito hipoglicemiante deste fruto amazônico, possibilitando ser explorado biotecnologicamente, para, por exemplo, controle do diabetes do tipo II. 


\section{AGRADECIMENTO}

Os autores deste artigo agradecem a Fundação de Amparo a Pesquisa do Estado do Amazonas (FAPEAM), ao Conselho Nacional de Desenvolvimento Científico e Tecnológico (CNPq) pelo financiamento recebido e a Coordenação de Aperfeiçoamento de Pessoal de Nível Superior (CAPES) pela bolsa concedida a SOBREIRA, C.R.C.

\section{REFERÊNCIA}

AGRA, M.F. et al. Synopsis of the plants know as medical poisonous in Northeast of Brazil. Revista Brasileira de Farmacognosia, v.17,p.114-40, 2007.

ALMEIDA, E.R. et al. Hypoglycemic effect of Bauhimia cheilandra in rats. Fitoterapia, v.77, p.276-8, 2006.

ASSOCIATION OF OFFICIAL ANALYTICAL CHEMISTS Official Methods of Analysis of AOAC Internacional, 17.ed. Gaithersburg, 2000.

BULLÓ, M. et al. Inflammation, obesity and comorbidities: the role of diet. Public Health Nutrition, v.10, p.1164-72, 2007.

CARVALHO, M.J. et al. Estudo farmacognóstico e atividade in vitro sobre a coagulação sanguínea e agregação plaquetária das folhas de Passiflora nítida Kunth (Passifloraceae). Acta Amazônica, v.40,n.1, p.199-206, 2010.

COLLAZOS, C. et al. La composición de los alimentos peruanos. Lima: Instituto de Nutrición, Ministerio de Salud. 1975. p.35.

CÓRDOVA, K.V. et al. Características físico-químicas da casca do maracujá amarelo (Passiflora edullis Flavicarpa Deneger) obtida por secagem. Boletim Centro de Pesquisa de Processamento de Alimentos, v.23, n.2, p.221-30, 2005.

DERIVI, S.C.N. et al. Efeito hipoglicemiante de rações à base de berinjela (Solanum melongena L.) em ratos. Ciência e Tecnologia de Alimentos, v.22, n.2, p.164-9, 2002. GONDIM, J.A.M. et al. Composição centesimal e de minerais em cascas de frutas. Ciência e Tecnologia de Alimentos, v.25, p.825-7, 2005.

HOWARTH, N.C. et al. Dietary fiber and weigth regulation. Nutrition Review, v.59, p.129-39, 2001.
INSTITUTO ADOLFO LUTZ - IAL. Normas analíticas do Instituto Adolfo lutz: métodos químicos e físicos de composição de alimentos, 3.ed. São Paulo. 1985.

KRAHN, C.L. et al. Avaliação do efeito da casca desidratada do maracujá (Passiflora edulis) e seu extrato aquoso na redução da glicemia em ratos diabéticos induzidos por aloxano. Revista Brasileira de Farmacologia, v.89, p.32-4, 2008.

MAIHARA, V.A. et al. Avaliação nutricional de dietas de trabalhadores em relação a proteínas, lipídios, carboidratos, fibras alimentares e vitaminas. Ciência e Tecnologia de Alimentos, v.26, n.3, p.672-7, 2006. MORAES, C.M. et al. Susceptibilidade de Passiflora nítida ao passion fruit woodness vírus. Fitopatologia Brasileira, v.27, p.108-13, 2002.

MORETTO, E. et al. Composição centesimal dos Produtos Alimentícios. In: MORETTO, E. et al. (Eds.). Introdução à ciência dos alimentos. Florianópolis: Editora da UFSC, 2002. p.19-56.

MIRA, G.S. et al. Visão retrospectiva em fibras alimentares com ênfase em beta-glucanas no tratamento do diabetes. Revista Brasileira de Ciências Farmacêuticas, v.45, p.11-20, 2009.

PI-SUNYER, X. Do glycemic index, glycemic load, and fiber play a role in insulin sensitivity, disposition index, and type 2 diabetes? Diabetes Care, v.28, p.2978-9, 2005. SCHOENFELDER, T. et al. Acute effect of Trema micrantha (Ulmaceae) on serum glucose levels in normal and diabetic rats. Journal of Ethnopharmacology, v.107, p.456-9, 2006.

SILVA, L.M.C. et al. Aposentados com diabetes tipo 2 na saúde da família em Ribeirão Preto, São Paulo-Brasil. Revista da Escola de Enfermagem da USP, v.44, n.2, p.462-8, 2010.

SOARES, J.C.M. et al. Níveis glicêmicos e de colesterol em ratos com Diabetes mellitus aloxano induzido, tratados com infusão de Bauhinia candicans ou Syzygium jambolaum. Ciência Rural, v.30, n.1, p.113118, 2000.

WEICKERT, M.O.; PFEIFFER, A.F.H. Metabolic effects of dietary fiber consumption and prevention of diabetes. American Society for Nutrition, v.138, p.439-42, 2008. WILD, S. et al. Global prevalence of diabetes: estimates for year 2000 and projections for 2030. Diabetes Care, v.31, n.3, p.596-615, 2004. 